\title{
Numerical study of gas-liquid nucleation in partially miscible binary mixtures
}

\author{
Pieter Rein ten Wolde and Daan Frenkel \\ FOM Institute for Atomic and Molecular Physics, Kruislaan 407, 1098 SJ Amsterdam, The Netherlands
}

(Received 21 July 1998; accepted 1 September 1998)

\begin{abstract}
We report a numerical study of homogeneous gas-liquid nucleation in a binary mixture. We study the size and the composition of the critical nucleus as a function of the composition and supersaturation of the vapor. As we make the (Lennard-Jones) mixture increasingly nonideal, we find that there is a regime where the critical nucleus is still miscible in all proportions, even though the bulk liquid phase is not. When these critical nuclei grow, their composition "bifurcates" to approach the value of one of the two bulk phases. For more strongly nonideal mixtures, the two species in the critical nucleus are no longer completely miscible: we observe droplets that are either rich in one species, or in the other. However, we do not find evidence for phase separation inside the critical nucleus - a scenario suggested by Talanquer and Oxtoby [J. Chem. Phys. 104, 1993 (1996)]. In fact, our simulations show that such demixed clusters have a higher free energy than critical nuclei that have an asymmetric composition. (C) 1998 American Institute of Physics. [S0021-9606(98)51246-9]
\end{abstract}

\section{INTRODUCTION}

Measurements of the rate of homogeneous gas-liquid nucleation provide increasingly precise and detailed information about the microscopic aspects of the nucleation process. ${ }^{1-8}$ From the measured nucleation rates, one can deduce the size and composition of the critical nuclei. ${ }^{9}$ Such information makes it possible to test nucleation theories in much more detail than was hitherto possible. The experiments indicate that classical nucleation theory (CNT) works fairly well for one-component systems of simple, nonpolar molecules. ${ }^{4,8}$ In particular, CNT predicts the size of critical nuclei surprisingly well. However, for binary systems the agreement between the experimental observations and the predictions of CNT is worse. ${ }^{2,3}$ For nonideal mixtures, CNT can even produce thermodynamic inconsistencies. Under certain conditions, CNT predicts that, at constant height of the nucleation barrier, the gas-phase activity of one component as a function of the activity of the other can have a positive slope. From the nucleation theorem ${ }^{9}$ it would then follow that one of the two components has a negative concentration in the critical nucleus. Laaksonen and Oxtoby, who used density functional theory to study nucleation in a mixture, ${ }^{10,11}$ argue that the failure of CNT can be traced back to the fact that this theory does not predict the composition of the critical nucleus correctly.

Talanquer and Oxtoby ${ }^{12}$ reported a more extensive study of the effect of composition and relative strength of the interactions on the nucleation behavior of a mixture. They found that, as expected, nearly ideal mixtures with positive (negative) deviations from ideal behavior require higher (lower) gas-phase activities for nucleation. However, for less ideal mixtures, they observed unexpected behavior. They found that in some cases, more than one type of critical nucleus could occur. In particular, they observed that nucleation of a mixture rich in the less volatile component can proceed via a nucleus that is poor in this component. More surprisingly, their theoretical analysis indicated that, under certain conditions, phase separation can take place inside the critical nucleus.

Computer simulation is a convenient tool to study the crucial entity in gas-liquid nucleation, namely the critical nucleus. Direct experimental observation of the critical nucleus is complicated by the fact that nucleation is both infrequent and fast: the chances of catching the system at the top of the nucleation barrier are slight. In computer simulation, it is possible to study the structure and free energy of the critical nucleus directly and in detail. This allows us to test the relevant theoretical predictions. In this paper we report a computer simulation study of gas-liquid nucleation in a binary mixture of Lennard-Jones particles. This is the same model system that was studied by Talanquer and Oxtoby. ${ }^{12}$

The rest of the paper is organized as follows: in the next section we describe the simulation techniques that we have used. We have developed a computational method that allows us to efficiently compare critical clusters that correspond to the same height of the nucleation barrier. As the rate of nucleation is dominated by the height of this freeenergy barrier, simulations at constant barrier height are comparable to experiments in which the size and composition of critical nuclei are often studied at a fixed nucleation rate.

\section{SIMULATION TECHNIQUES}

\section{A. Cluster free energy}

At a given pressure, temperature, and composition of the vapor, the excess free energy of a nucleus depends both on its size and on its composition. The method that we used to compute the free energy of an arbitrary liquid cluster is described in detail in Ref. 13. Below, we briefly summarize our approach.

In gas-liquid nucleation, the density of liquid clusters is 
usually so low that the interactions between them can be neglected. Then, the average number $N_{n}$ of liquid clusters of size $n$ is given by ${ }^{13}$

$$
\left\langle N_{n}\right\rangle=\exp \left[-\beta\left(F_{n}-n \mu\right)\right]=\exp [-\beta \Delta F],
$$

where $F_{n}$ is the free energy of a cluster of size $n, \mu$ is the chemical potential of particles in the vapor, and $\beta \equiv 1 / k_{B} T$ is the reciprocal temperature with $k_{B}$ Boltzmann's constant and $T$ the absolute temperature. In order to arrive at the above expression, we only ignore the interaction between clusters, not the interaction of the cluster with the surrounding vapor. Clearly, in order to compute the properties of a cluster, we need to be able to identify a cluster unambiguously. In the present study we have used the same cluster criterion that was used in Ref. 13. This criterion distinguishes between liquidlike and vaporlike particles, on the basis of the local density around a particle. Neighboring liquidlike particles that are within a certain cut-off distance are said to be connected. A liquid cluster consists of all liquidlike particles that are connected. We stress that our cluster criterion is only sensitive to the size of the cluster and not to its composition. We therefore do not impose a particular cluster composition.

The number of clusters, denoted by $N_{n}$, is an extensive quantity, i.e., it is proportional to the size of the system. It is useful to relate the number of clusters to the total number of particles in the system, denoted by $N$, via a probability $P_{n}$ that is intensive,

$$
P_{n} \equiv \frac{N_{n}}{N} .
$$

This, in turn, defines an intensive isobaric-semi-grand (see below) free-energy of the cluster,

$$
\Delta Y \equiv-k_{B} T \ln \left[P_{n}\right] .
$$

The average number of clusters is then given by

$$
\left\langle N_{n}\right\rangle=N \exp [-\beta \Delta Y] \text {. }
$$

The probability distribution function $P_{n}$ is an equilibrium quantity and can be computed both by molecular dynamics (MD) and by Monte Carlo (MC). However, the brute force approach, in which we would simulate the metastable vapor, and simply count the number of liquid clusters, will not work. At the moderate degree of supersaturation that is characteristic for most experimental studies of gas-liquid nucleation, the height of the barrier is typically of the order of $75 k_{B} T$. This implies that, even if the number of particles in the system is equal to Avogadro's number, the probability of finding a critical nucleus at any given instant is of the order of one in a billion. In most simulations, the number of particles is some 20 orders of magnitude less, and so is the probability of finding a critical nucleus in a simulation. Hence, standard MD or MC simulations are ill-suited to study the formation of critical nuclei. In order to obtain good statistics near the top of the nucleation barrier, we have employed the umbrella sampling technique. ${ }^{14}$ The basic idea behind this approach is that we can influence the frequency with which a particular range of cluster sizes is sampled during a simulation by adding a fictitious biasing potential to the Hamiltonian of the model system. Subsequently, we correct for the bias that we have introduced in the sampling. ${ }^{14}$

\section{B. Contour of constant barrier height}

Using the umbrella sampling technique, we can stabilize not only critical but also precritical (and postcritical) nuclei. In this way we can obtain information about the structure and free energy of these nuclei. In the present study we focus our attention mainly on the critical clusters, i.e., nuclei at the top of the free-energy barrier. At constant temperature, the height of the free-energy barrier depends both on the composition and the pressure of the vapor phase. However, as the nucleation rate is a very steep function of the barrier height, most experimental studies focus effectively on a narrow "window" of barrier heights. For this reason, we also look in our numerical simulation at the composition dependence of critical nuclei at constant barrier height. To achieve this, we adjust the pressure for every composition such that the barrier height is kept fixed at a given reference value. In principle, we could compute the full free-energy barrier to nucleation by the umbrella sampling technique for a series of pressures, in order to find the desired pressure for a given composition. However, even for only one composition and one pressure, computing the full free-energy curve is already quite time consuming. We therefore follow a different approach, which is close in spirit to the Gibbs-Duhem integration method to trace phase-coexistence curves. ${ }^{15}$

We first compute the nucleation barrier for one pure component at a given pressure, using umbrella sampling. We then increase the activity fraction of the other component. In order to keep the height of the nucleation barrier constant, we have to adjust the pressure. Information about the required change in pressure can be obtained by simulating the system not only at the top of the barrier, but also in the metastable vapor phase. For both states we can determine how the free energy varies with the activity fraction and pressure. By setting the variation in free energy for both states equal, thus keeping the height of the barrier constant, we get a Clapeyron-type of equation which yields a relation between the change in pressure with the change in activity fraction.

Let us make this approach more explicit. It will be convenient to work in the (isobaric) semigrand ensemble. The isobaric semigrand free energy $Y$ is the Legendre transform of the Gibbs free energy $G$, which, for a binary system, is a function of the total number of particles $N$, the number of particles of species $2, N_{2}$, the pressure $P$, the temperature $T$, and the order parameter $n$. The first differential of the Gibbs free energy is

$$
\begin{aligned}
d G\left(N, N_{2}, P, T, n\right)= & \mu_{1}(n) d N+\left(\mu_{2}(n)-\mu_{1}(n)\right) d N_{2} \\
& +V(n) d P-S(n) d T+\left.\frac{\partial G}{\partial n}\right|_{N, N_{2}, P, T} d n,
\end{aligned}
$$

where $\mu_{i}$ is the chemical potential of species $i, V$ is the 
volume, and $S$ is the entropy of the system. Now the semigrand ensemble is obtained by a Legendre transformation between the variables $N_{2}$ and $\left(\mu_{2}-\mu_{1}\right)$,

$$
Y\left(N, \mu_{2}-\mu_{1}, P, T, n\right)=G\left(N, N_{2}, P, T, n\right)-N_{2}(n)\left(\mu_{2}-\mu_{1}\right) \text {, }
$$

or, in differential form,

$$
\begin{aligned}
d Y\left(N, \mu_{2}-\mu_{1}, P, T, n\right)= & \mu_{1}(n) d N-N_{2}(n) d\left(\mu_{2}-\mu_{1}\right) \\
& +V(n) d P-S(n) d T \\
& +\left(\frac{\partial Y}{\partial n}\right)_{N, P, T, \mu_{2}-\mu_{1}} d n .
\end{aligned}
$$

At constant temperature and number of particles, Eq. (7) reduces to

$$
\begin{aligned}
& d Y\left(N, P, T, \mu_{2}-\mu_{1}, n\right) \\
& \quad=V(n) d P-N_{2}(n) d \Delta \mu+\left(\frac{\partial Y}{\partial n}\right)_{N, P, T, \Delta \mu} d n,
\end{aligned}
$$

where $\Delta \mu=\mu_{2}-\mu_{1}$. In order to keep the height of the barrier constant, the variation in the free energy at the top of the barrier, whose position is denoted by $n^{*}$, should be equal to the variation in the free energy in the metastable vapor phase, with $n=0$. In general, when the activity fraction and the pressure are changed, the top of the barrier can change. However, at the top of the barrier, the partial derivative of the free energy with respect to cluster size, $\partial Y(n) / \partial n$, is zero, so that the last term in Eqs. (7) and (8) drops out. In the metastable vapor $n$ is zero and remains zero. Hence, the last term is zero, also for the vapor phase. Equation (8) then reduces to

$$
\begin{aligned}
& d Y\left(n^{*}\right)=d Y(0) ; \\
& V\left(n^{*}\right) d P-N_{2}\left(n^{*}\right) d \Delta \mu=V(0) d P-N_{2}(0) d \Delta \mu,
\end{aligned}
$$

from which we obtain

$$
\left(\frac{d P}{d \Delta \mu}\right)^{*}=\frac{N_{2}\left(n^{*}\right)-N_{2}(0)}{V\left(n^{*}\right)-V(0)},
$$

where the asterisk denotes differentiation at constant barrier height. The chemical potential difference $\Delta \mu$ can be written in terms of the activities of species 1 and 2 ,

$$
\Delta \mu=k_{B} T \log \left(\frac{a_{2}}{a_{1}}\right),
$$

where $a_{i}=\exp \left[\beta \mu_{i}\right]$ denotes the activity of species $i$. We can rewrite the above expression as

$$
\Delta \mu=k_{B} T \log \left(\frac{x_{a_{2}}}{1-x_{a_{2}}}\right),
$$

where $x_{a 2}$ is the activity fraction of species 2 , defined as

$$
x_{a_{2}} \equiv \frac{a_{2}}{a_{1}+a_{2}} .
$$

We thus obtain the following expression for the variation of the pressure with activity fraction

$$
\left(\frac{d P}{d x_{a_{2}}}\right)^{*}=\frac{k_{B} T}{x_{a_{2}}\left(1-x_{a_{2}}\right)} \frac{N_{2}\left(n^{*}\right)-N_{2}(0)}{V\left(n^{*}\right)-V(0)} .
$$

To compute a contour of constant barrier height on the freeenergy barrier surface, we have to integrate the above expression in the $P, x_{a_{2}}$ plane by computing $N_{2}$ and $V$, both at the top of the barrier and in the metastable vapor. We have integrated Eq. (15) by a fourth-order predictor-corrector scheme. We start with $x_{a_{2}}=0$, which corresponds to the limit that only one species is present. At $x_{a_{2}}=0$, the value of $\left(d P / d x_{a_{2}}\right)$ is undefined [see Eq. (15)] because both the numerator $\left[N_{2}\left(n^{*}\right)-N_{2}(0)\right]$ and the denominator $\left[x_{a_{2}}(1\right.$ $\left.\left.-x_{a_{2}}\right)\right]$ vanish for $x_{a_{2}}=0$. However, $\left(d P / d x_{a_{2}}\right)$ itself is finite. To bootstrap the integration procedure we have adopted the scheme of Bolhuis and Frenkel. ${ }^{16}$ Instead of calculating successive points step by step, we "guess" the first points on the $P, x_{a_{2}}$ contour. For every point we perform a simulation (or actually two, one in the vapor and one at the top of the barrier) to compute the derivative of $P$ with respect to $x_{a_{2}}$ according to Eq. (15). Subsequently we fit the derivatives to a polynomial in $x_{a_{2}}$. The polynomial is integrated to give new pressures which are then used in the next iteration. We repeat this procedure until convergence of the pressure is reached. After we have generated the first few points on the contour in this way, we use these points to start the fourthorder predictor-corrector scheme to obtain the rest of the contour.

To compute the derivative in Eq. (15) we need to know the position of the top of the barrier with a high accuracy, as $N_{2}(n)$ and $V(n)$ strongly depend on $n$. For every pressure and activity, we therefore perform not one, but three simulations near the top of the barrier: one at the estimated top, and one on either side. The histogram of cluster sizes near the top of the barrier is fitted to a polynomial to obtain the variation of the free energy with cluster size [via Eq. (3)]. This allows us to locate the position of the top of the barrier. Next, to get the volume, $V\left(n^{*}\right)$ and the number of particles of species 2 , $N_{2}\left(n^{*}\right)$, at the top of the barrier, we fit the histograms of $V(n)$ and $N_{2}(n)$ to polynomials and insert the value of the critical droplet size into these polynomial expressions.

\section{THE SYSTEM}

We studied a simple model for a binary mixture, namely one in which the particles interact via the Lennard-Jones pair potential

$$
v_{i j}(r)=4 \epsilon_{i j}\left[\left(\frac{\sigma_{i j}}{r}\right)^{12}-\left(\frac{\sigma_{i j}}{r}\right)^{6}\right],
$$

where $r$ is the interparticle distance, $\epsilon_{i j}$ is the Lennard-Jones well depth corresponding to the interaction between species $i$ and $j$, and $\sigma_{i j}$ is the corresponding Lennard-Jones diameter. Following Talanquer and Oxtoby ${ }^{12}$ we take $\sigma_{11}=\sigma_{22}=\sigma_{12}$ $=\sigma$.

The structure and composition of our nuclei are then determined by the values of the "volatility parameter" 


$$
\epsilon_{22}^{*}=\frac{\epsilon_{22}}{\epsilon_{11}}
$$

and the "mixing parameter"

$$
\Lambda *=\frac{\Lambda}{\epsilon_{11}}=\frac{\epsilon_{11}+\epsilon_{22}-2 \epsilon_{12}}{\epsilon_{11}} .
$$

The volatility parameter $\epsilon_{22}^{*}$ measures the relative volatility of the two components, and the mixing parameter $\Lambda^{*}$ determines the energy of mixing particles of different species. Note that $\Lambda^{*}$ does not measure the deviation from the Lorentz-Berthelot mixing rule $\left(\epsilon_{12}=\sqrt{\epsilon_{11} \epsilon_{22}}\right)$. In fact, when $\epsilon_{12}=\sqrt{\epsilon_{11} \epsilon_{22}}, \Lambda *>0$, unless $\epsilon_{11}=\epsilon_{22}$.

In most nucleation experiments ${ }^{3-5}$ the volume is fixed. This means that the nucleation of a liquid droplet leads to a decrease in vapor pressure, and in general, also to a change in the composition of the vapor phase. However, as already indicated, the concentration of critical nuclei is usually so small that the change in pressure and composition is negligible. That is, when a critical nucleus is formed, the activities of the species in the vapor phase are unaltered. In principle, we could simulate the experimental situation by performing a NVT simulation. However, a large excess number of particles would then be required in order to keep the vapor phase activities constant. It is therefore much more natural to work in the isobaric-semi-grand $(N P T \Delta \mu)$ ensemble or in the grand-canonical $(\mu V T)$ ensemble. In both ensembles a liquid droplet can be formed at constant chemical potentials of the vapor species without actually having to simulate an excessive number of vapor particles. In the grand-canonical ensemble this is accomplished by the insertion and removal of particles. However, at high densities the insertion probability can be too low to obtain reasonable statistics. It is then more convenient to work in the isobaric-semi-grand ensemble. In this ensemble, the volume of the system is adjusted such that the pressure in the vapor surrounding the liquid drop is maintained at a constant value, irrespective of the size of the liquid cluster. ${ }^{13}$ In addition, in a semigrand Monte Carlo simulation, the composition in the vapor is kept constant, by allowing for Monte Carlo moves that swap the identity of particles (see, e.g., Ref. 17). Hence, this approach effectively mimics the experimental situation in which the gas-phase activities are constant, but it avoids the insertion and removal of particles that is required in the grandcanonical ensemble. More details about the Monte Carlo scheme that is used to study (pre) critical nuclei can be found in Ref. 13.

The number of particles in all simulations was $N=864$. As the size of the largest critical cluster in our simulations was fewer than 600 particles and the density of the surrounding vapor is very low, the system size was always sufficiently large that system-size artifacts can be excluded. We have truncated the potential at $r_{c}=2.5 \sigma$ and shifted the potential such that it is zero at the cutoff. No long-range corrections were made. We applied cubic periodic boundary conditions. In what follows, we use reduced units, such that $\epsilon_{11}$ is the unit of energy and $\sigma$ is the unit of length.

\section{RESULTS AND DISCUSSION}

In order to facilitate comparison of our findings with recent experiments ${ }^{2-5}$ and theories ${ }^{12}$ on nucleation in binary systems, we will use, as much as possible, the same notation as employed in these publications. In these studies the "onset activities" $a_{i 0}=\exp \left[\beta \mu_{i 0}\right]$ are defined as those activities for which the nucleation rate (in experiments) or the height of the barrier (in theory) is kept at a fixed reference value. As we do not determine the nucleation rate itself, but the height of the nucleation barrier, we follow Talanquer and Oxtoby ${ }^{12}$ and define the onset activities as those activities for which the height of the barrier is constant. This seems reasonable, as the rate of nucleation depends strongly on the barrier height, and only weakly on the kinetic prefactor. We can therefore expect that the onset activities follow the same behavior as the experimentally determined onset activities that correspond to a fixed nucleation rate.

The composition of the clusters is usually expressed in terms of the normalized activity fraction of one of the components. The normalized activity fraction of component 2 is defined as

$$
x_{a_{2 n}} \equiv \frac{a_{2 n}}{a_{1 n}+a_{2 n}},
$$

where the normalized onset vapor activities $a_{1 n}$ are given by

$$
a_{i n} \equiv a_{i 0} / a_{i 0}^{0}
$$

and $a_{i 0}^{0}$ represents the onset activity for the pure vapor of component $i$.

We performed two sets of simulations. The first set corresponds to asymmetric mixtures, for which $\epsilon_{22}^{*}=1.1$. The second corresponds to symmetric mixtures with $\epsilon_{22}^{*}=1.0$. The starting point for all simulations was the one-component system with $x_{a_{2}}=0.0$, i.e., a system consisting of pure species 1 . In Ref. 13 we computed the height of the nucleation barrier in this system for $T=0.741$ [which is $32 \%$ below the critical temperature $T_{c}=1.085$ (Ref. 18)] and $P=0.01202$, which corresponds to a supersaturation $S=1.53$. At this degree of undercooling, the height of the nucleation barrier is $56.7 k_{B} T$ (Ref. 13). In the present simulations, we keep the temperature constant, but vary the activity fraction and pressure according to Eq. (15). In this way, we ensure that the height of the barrier remains constant. Hence, all results that we present below refer to a reference barrier height $\Delta Y_{0}^{*}$ $=56.7 k_{B} T$.

\section{A. Asymmetric mixtures}

We first discuss the behavior of the asymmetric mixtures, as they provide a good test for the integration scheme discussed in Sec. II B. The volatility parameter is $\epsilon_{22}^{*}=1.1$. The fact that $\epsilon_{22}^{*}>1$ means that component 1 is the more volatile component. We studied asymmetric mixtures with this value of the volatility parameter for three different values of the mixing parameter $\Lambda^{*}: \Lambda^{*}=0.1,0.3$, and 0.5 . As the volatility parameter is held constant, the two onecomponent limits, with $x_{a_{2 n}}=0.0$ and $x_{a_{2 n}}=1.0$, are the same for all three values of $\Lambda^{*}$. This can be used as a test for the accuracy of the integration scheme discussed in Sec. II B: 


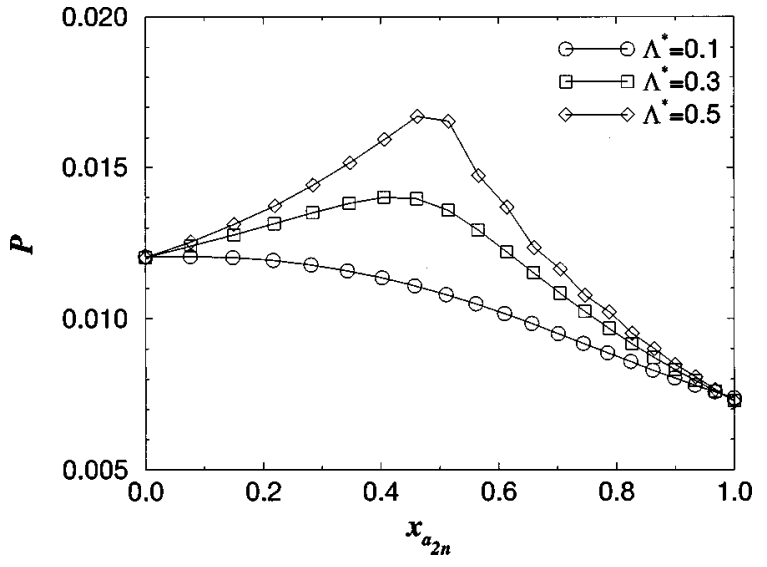

FIG. 1. The reduced pressure $P$ as a function of the normalized activity fraction $x_{a_{2 n}}$ for an asymmetric mixture with $\epsilon_{22}^{*}=1.1$ for three different values of the mixing parameter $\Lambda^{*}$. All curves correspond to a reference work of formation of $\Delta Y_{0}^{*}=56.7 k_{B} T$. In this figure and all subsequent figures, we use $\sigma_{11}=\sigma_{22}=\sigma_{12}$ as our unit of length and $\epsilon_{11}$ as our unit of energy. In all cases, $k_{B} T / \epsilon_{11}=0.741$.

Although we do not know beforehand what value of the pressure is required to get the reference barrier height $Y_{0}$ $=56.7 k_{B} T$ in the limit $x_{a_{2 n}}=1.0$, we do know that this pressure cannot depend on $\Lambda^{*}$. Figure 1 shows the pressure as a function of the activity fraction for the three different mixtures. It is seen that at intermediate values of the activity fraction, the pressures are different for the different types of mixtures. However, in the one-component limits the pressures are the same $\left[P\left(x_{a_{2 n}}=1.0\right)=0.00730 \pm 0.00007\right]$. This means that the integration scheme is not only an efficient, but also an accurate method to compute contours of constant free energy on the free-energy barrier surface.

Figure 1 shows that, as the activity fraction is increased from zero, the pressure initially increases. In other words, mixing inhibits the nucleation process, and the higher the value of the mixing parameter, the larger the increase in pressure. At some point, however, the pressures goes down again as the nuclei become enriched in the less volatile component. This is illustrated in Fig. 2, where we show the ex-

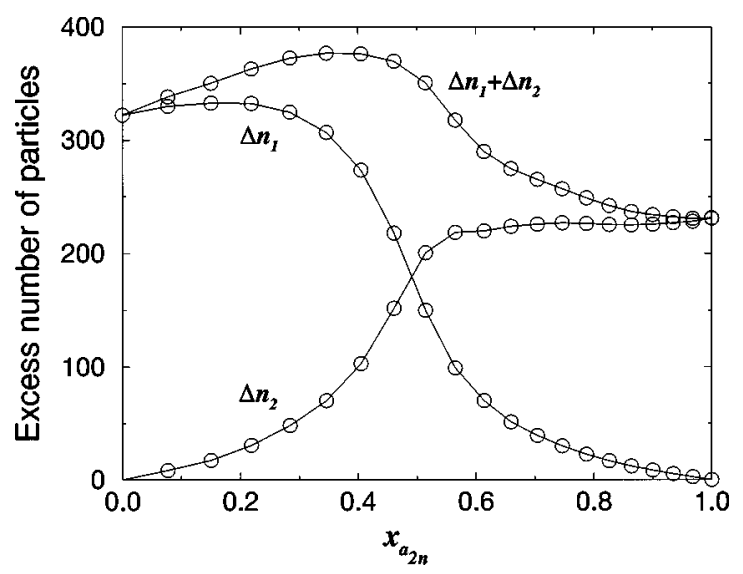

FIG. 2. Size and composition of the critical nuclei as a function of the normalized activity fraction $x_{a_{2 n}}$ in a binary system with $\epsilon^{*}=1.1$ and $\Lambda^{*}$ $=0.3$, corresponding to a nucleation barrier of height $\Delta Y_{0}^{*}=56.7 k_{B} T . \Delta n_{1}$ and $\Delta n_{2}$ are the excess numbers of particles of species 1 and 2, respectively. cess number of particles of species 1 and 2 as a function of the activity fraction for $\Lambda^{*}=0.3$. The excess number of particles of species $i$ is defined as

$$
\Delta n_{i}=4 \pi \int_{0}^{\infty}\left(\rho_{i}(r)-\rho_{v, i}\right) r^{2} d r
$$

where $\rho(r)_{i}$ is the density of species $i$ in a spherical shell of radius $r$ around the center of mass of the cluster and $\rho_{v, i}$ is the density of species $i$ in the vapor. The excess number of particles in a critical nucleus is a quantity that can be deduced from experiments using the nucleation theorem. ${ }^{9}$

In the limit $x_{a_{2 n}}=0.0$, the critical nucleus contains some 325 particles. When the activity fraction is increased, the concentration of species 2 increases. But as mixing of the two species is unfavorable, the relative concentration of species 2 in the cluster is initially less than in the vapor phase. Moreover, the total number of particles in the critical nucleus increases with $x_{a_{2 n}}$. However, at an activity fraction of $x_{a_{2 n}}$ $=0.4$, component 2 begins to dominate the nucleation process and the fraction of this species in the critical nucleus rapidly increases. At the same time, the total number of particles in the cluster starts to decrease. At $x_{a_{2 n}}=1.0$ (pure species 2), the critical cluster size is 235 particles. Note that this cluster size is smaller than the critical nucleus size at $x_{a_{2 n}}=0.0$. This is due to the fact that species 2 is less volatile than species 1 .

Let us next examine the structure of the critical nuclei. Clarke et al. ${ }^{19}$ reported a computer simulation study of binary liquid Lennard-Jones clusters in vacuo. The simulations of Ref. 19 were performed at a reduced temperature $T$ $=0.31$, which is less than half the temperature that we imposed during our simulations. Clarke et al. found that for $\epsilon_{22}^{*} \neq 1.0$ and $\Lambda^{*}>0.0$, spherical clusters can occur that consist of a core rich in the less volatile component, coated by a shell rich in the more volatile component. Such behavior is to be expected on the basis of macroscopic (wetting) arguments. However, under the conditions studied in our simulations, we find little evidence for the existence of such compositional inhomogeneities. In almost all clusters that we studied, the two species appeared well mixed. Only for the critical nuclei at $\Lambda^{*}=0.5$ and $x_{a_{2 n}}=0.52$ do we find that the concentration of species 1 (the more volatile component) is significantly higher in the surface than in the core of the droplet (see Fig. 3).

\section{B. Symmetric mixtures}

We studied symmetric mixtures, for which $\epsilon_{22}^{*}=1.0$, for the following values of the mixing parameter $\Lambda^{*}: \Lambda^{*}$ $=-0.1,0.1,0.3,0.5$, and 0.7. The symmetric mixture with $\Lambda^{*}=0.0$ corresponds to an ideal mixture, that is, $\epsilon_{11}=\epsilon_{22}$ $=\epsilon_{12}$. We will use this "mixture" as a reference. The mixtures for $\Lambda^{*} \leqslant 0.3$ are weakly nonideal and the species are fully miscible in the bulk liquid. However, for $\Lambda^{*}>0.3$, the species demix in the bulk. Below, we first discuss the weakly nonideal mixtures and subsequently the mixtures that show a macroscopic miscibility gap. 


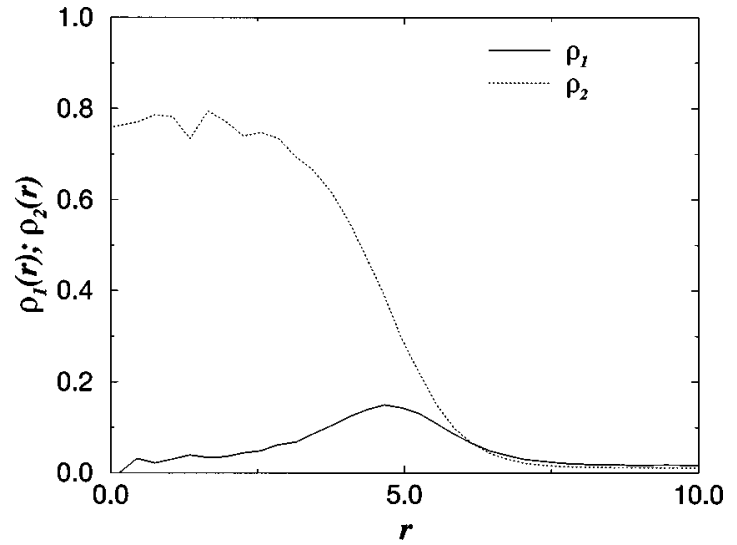

FIG. 3. Radial density profile of species 1 and 2 for a critical nucleus in a system with $\epsilon_{22}^{*}=1.1$ and $\Lambda^{*}=0.5$. The normalized activity fraction is $x_{a_{2 n}}=0.52$ and the height of the nucleation barrier is $\Delta Y_{0}^{*}=56.7 k_{B} T$. Units as in Fig. 1.

\section{Weakly nonideal liquid mixtures}

The weakly nonideal liquid mixtures do not show bulk liquid-liquid phase separation and, not surprisingly, the two species are also fully mixed in the critical nuclei. However, depending on the sign of $\Lambda^{*}$, nucleation is either enhanced or hindered by mixing. Figure 4 shows the pressure as a function of the activity fraction. Note that as species 1 and 2 have identical properties, the figure is symmetric around $x_{a_{2 n}}=0.5$. For comparison, we also show the contour (a straight line) for the ideal mixture. For negative values of $\Lambda^{*}$, mixing of the two species in the critical cluster lowers the pressure that is needed to maintain a constant barrier height. In other words, mixing enhances the nucleation process. For positive values of $\Lambda^{*}$ the vapor pressure has to be increased in order to keep the height of the barrier constant.

This behavior can be understood by examining the compositions of the critical nuclei. Figure 5 shows the excess number of particles of species 1 and 2 as a function of the activity fraction for $\Lambda^{*}=-0.1$ and $\Lambda^{*}=0.1$. Again, we also show the (trivial) result for the ideal mixture. For such a

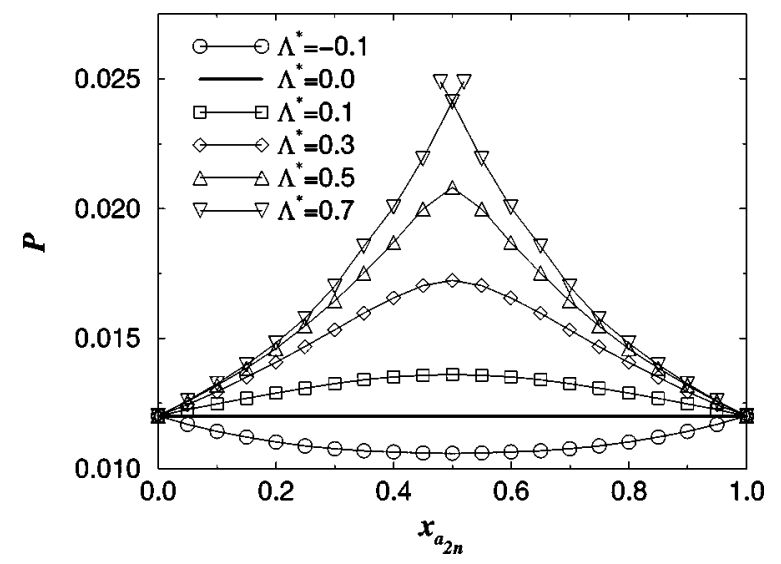

FIG. 4. The reduced pressure $P$ as a function of the normalized activity fraction $x_{a_{2 n}}$ for symmetric mixtures $\left(\epsilon_{22}^{*}=1.0\right)$ with different values of the mixing parameter $\Lambda^{*}\left(\Delta Y_{0}^{*}=56.7 k_{B} T\right)$. The curve for the ideal mixture with $\epsilon_{22}^{*}=1.0$ and $\Lambda^{*}=0.0$ is indicated by the straight solid line. Units as in Fig. 1.

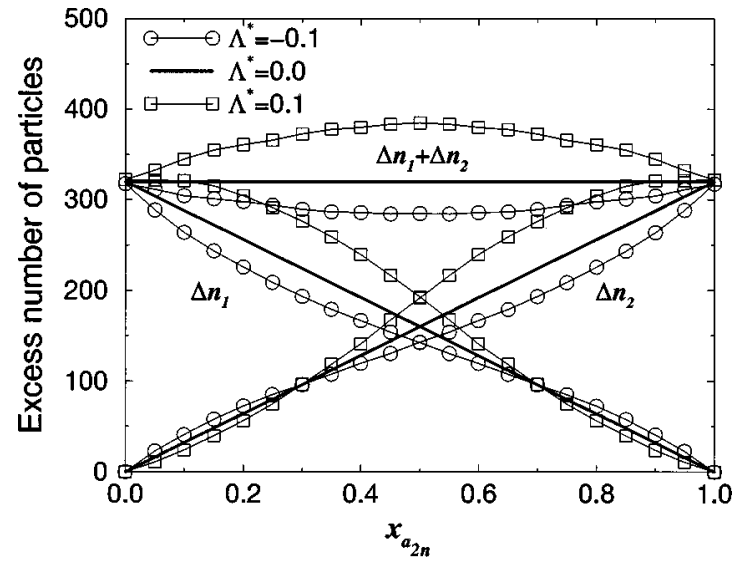

FIG. 5. The excess number of particles of species 1 and 2 (as well as their sum) in critical nuclei as a function of the normalized activity fraction $x_{a_{2 n}}$ for three symmetric mixtures that differ in the value of $\Lambda^{*}$. The ideal mixture with $\epsilon_{22}^{*}=1.0$ and $\Lambda^{*}=0.0$ is indicated by the straight solid lines and the onset work of formation is $\Delta Y_{0}^{*}=56.7 k_{B} T$.

mixture, both the composition of the critical nucleus and that of the vapor phase are equal to the activity fraction. Moreover, for an ideal mixture the size of the critical nucleus does not depend on composition.

For negative values of $\Lambda^{*}$, the critical-nucleus size shrinks when the species are mixed. Moreover, critical nuclei are enriched in the minority component when compared to the ideal mixture. For positive values of $\Lambda^{*}$, the opposite behavior is found. This can be seen more clearly in Fig. 6, which shows the molar fraction $x_{2}$ of species 2 in the critical nucleus as a function of the activity fraction $x_{a_{2 n}}$ (i.e., the composition in the dilute vapor).

\section{Demixing transition}

Let us now consider the more strongly nonideal mixtures that have a tendency to demix in the bulk, i.e., mixtures with large positive values of $\Lambda^{*}$.

When $\Lambda^{*}$ is increased, the critical clusters become increasingly enriched in the majority component as compared

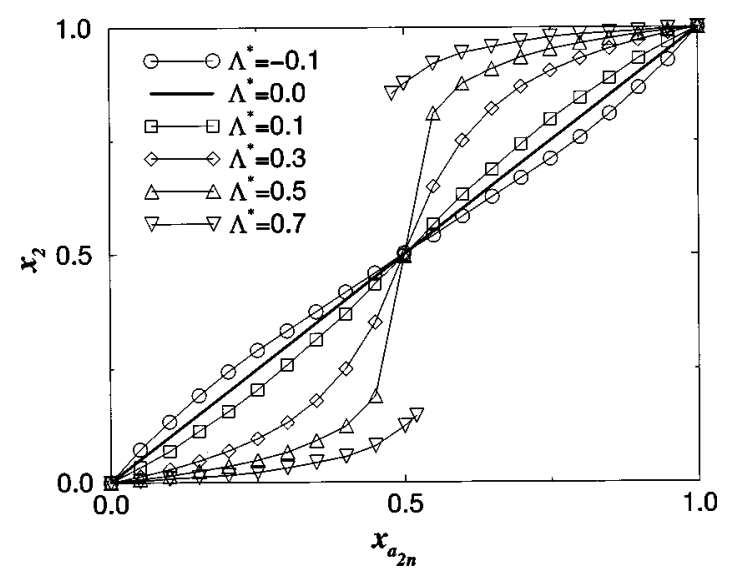

FIG. 6. Cluster composition $x_{2}$ as a function of the normalized activity fraction $x_{a_{2 n}}$ for (symmetric) mixtures with $\epsilon_{22}^{*}=1.0$ and an onset work of formation $\Delta Y_{0}^{*}=56.7 k_{B} T$. The ideal mixture with $\epsilon_{22}^{*}=1.0$ and $\Lambda^{*}=0.0$ is indicated by the thick solid line. 


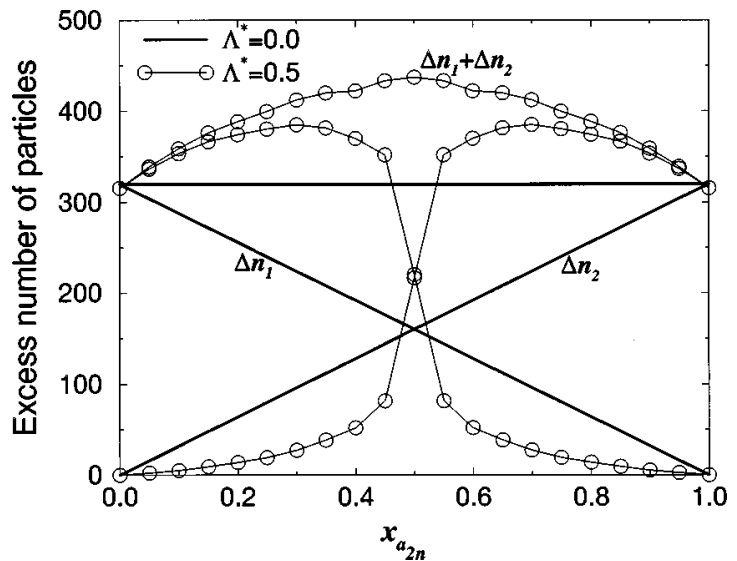

FIG. 7. Size and composition of the critical nuclei as a function of the normalized activity fraction $x_{a_{2 n}}$ for a symmetric mixture $\left(\epsilon_{22}^{*}=1.0\right)$ with $\Lambda^{*}=0.5\left(\Delta Y_{0}^{*}=56.7 k_{B} T\right)$. For comparison we also indicate the composition of the ideal mixture with $\epsilon_{22}^{*}=1.0$ and $\Lambda^{*}=0.0$.

to the ideal mixture. Figure 7 shows the composition of the critical cluster as a function of the activity fraction for $\Lambda^{*}$ $=0.5$. It is seen that up to an activity fraction of $x_{a_{2 n}}=0.5$, the cluster almost exclusively consists of species 1 , whereas for an activity fraction $x_{a_{2 n}}>0.5$ it consists almost only of species 2. Still, for $x_{a_{2 n}}=0.5, x_{2}=0.5$. That is, at $x_{a_{2 n}}$ $=0.5$, both species are present in equal amounts in the critical cluster. Yet, at $\Lambda^{*}=0.5$, the bulk liquid already exhibits phase separation. We thus have a situation in which the species are mixed in the cluster, but phase separated in the bulk liquid. A natural question to ask is therefore the following: how do these critical nuclei develop into a bulk liquid?

To answer this question, we have computed the free energy as a function of the composition for clusters of different size, at $\Lambda^{*}=0.5$ and $x_{a_{2 n}}=0.5$. We have studied a precritical cluster of 255 particles, a precritical cluster of 368 particles, a cluster near the top of the barrier of 472 particles, and a postcritical cluster of 571 particles. Figure 8 shows the free

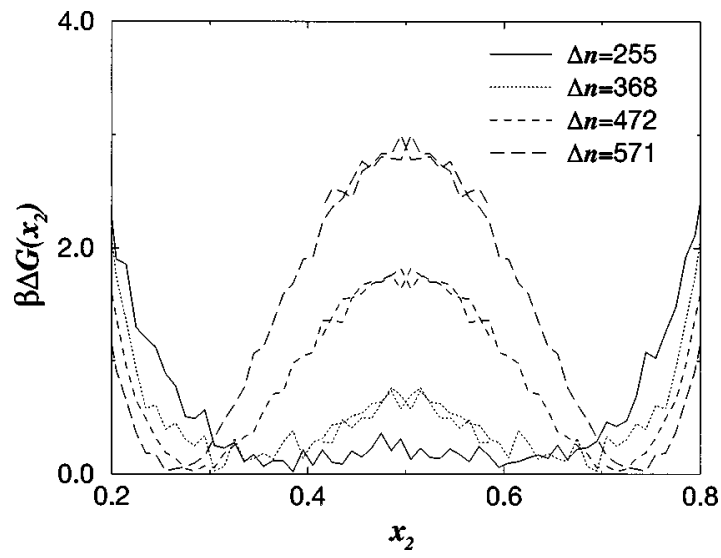

FIG. 8. Excess free energy as a function of the composition $x_{2}$ for nuclei of different size in a system with $\epsilon_{22}^{*}=1.0$ and $\Lambda^{*}=0.5$. The activity fraction is $x_{a_{2 n}}=0.5$ and the imposed pressure $P=0.021$, corresponding to a barrier height of $\Delta Y_{0}^{*}=56.7 k_{B} T$. The size of the critical cluster, i.e., the cluster at the top of the free-energy barrier, is around 440 particles. In order to facilitate the comparison of the free-energy curves we have set the free energy of the minima to zero. energy as a function of composition $x_{2}$ for these clusters. It is seen that the free-energy curve of the cluster of 255 particles only shows one broad minimum at $x_{2}=0.5$. However, for a cluster size of around 368 particles, the free-energy curve starts to develop two minima, centered at $x_{2}=0.3$ and $x_{2}$ $=0.7$, separated by a small free-energy barrier at $x_{2}=0.5$. For larger cluster sizes, the minima shift to the onecomponent limits and the free-energy barrier separating the two minima increases. As the mixture is symmetric, the freeenergy curves are symmetric around $x_{2}=0.5$.

The important point to note is that the saddle point that separates the stable liquid from the metastable vapor does not correspond to a critical cluster in which both species are mixed and equally present. Rather, there are two saddle points of equal free energy. Both saddle points correspond to a critical cluster of around 440 particles, but at one the cluster is of composition $x_{2}=0.3$, whereas the composition in the cluster at the other saddle point is $x_{2}=0.7$. However, the free-energy barrier separating the two saddle points is still so low that the critical cluster can easily jump back and forth between them.

As the critical nucleus evolves into a postcritical nucleus, there comes a point where the free-energy barrier that separates the two minima becomes too large for the cluster to cross. Two "channels" in the free-energy landscape have developed, one leading to a bulk liquid rich in species 1 , the other to a bulk liquid rich in species 2. Although both pathways for the formation of the bulk liquid are equally probable and both bulk liquid phases are equally likely to occur, once the system has chosen either path, it has to follow that path-it cannot make the transition to the other channel any more.

Yet, because rapid interconversion between the two critical nuclei is possible, the nucleation theorem would indicate a 50-50 composition of the critical nucleus. Clearly, this refers to the average composition of the critical nucleus. In particular, a macroscopic droplet of phase 1 may well have evolved from a critical nucleus that was rich in species 2 . Hence, this implies that in experiments on nucleation in moderate nonideal mixtures, the nucleation theorem will not reveal the true saddle point in the nucleation pathway. We note here that a similar conclusion was drawn by Oxtoby and Laaksonen ${ }^{20}$ who pointed out that caution is required in applying the nucleation theorem in the case of partially miscible mixtures. Recent experiments by Viisanen et al. on nucleation in binary mixtures of nonane with short-chain alcohols, may well belong to this category. ${ }^{5}$ In fact, it would be interesting if the nucleation rates of the two phases of the bulk liquid could be measured independently: if the "bifurcation" in the free-energy landscape occurs after the critical nucleus is formed, then the nucleation theorem will reveal the same critical nucleus for both liquids. However, if there is a high free-energy barrier between the critical nuclei that evolve into the two bulk phases, then the two nucleation rates will reveal the existence of two distinct critical nuclei. In our model system, this would be the case for $\Lambda^{*}=0.7$.

For $\Lambda^{*}=0.7$ and $x_{a_{2 n}}=0.5$, the path for the formation of the bulk liquid bifurcates for clusters that are appreciably smaller than the critical nucleus. We now have two different 
types of critical nuclei, one rich in component 1 and the other rich in component 2. At $x_{a_{2 n}}=0.5$, the two types of critical nuclei are of equal free energy and are formed with equal probability. However, once the critical nucleus of either type is formed, it cannot transform into the other type, due to the large free-energy barrier separating the two saddle points. Furthermore, the dependence of both the pressure and the cluster composition $x_{2}$ on the activity fraction $x_{a_{2 n}}$ for $\Lambda^{*}=0.7$ exhibits hysteresis at $x_{a_{2 n}}=0.5$, as can be seen in Figs. 4 and 6, respectively. This is due to the free-energy barrier separating the two types of nuclei. Only at $x_{a_{2 n}}$ $=0.52$ (or $x_{a_{2 n}}=0.48$ ) the height of the barrier is sufficiently reduced that the transition from one type of cluster to the other can take place on the time scale of a simulation.

The possibility of the simultaneous occurrence of two types of critical clusters was predicted by Talanquer and Oxtoby, ${ }^{12}$ using density functional theory, and by Ray et $a .^{21}$ using classical nucleation theory. The present simulations confirm this prediction. Of course, as the simulations illustrate, only at $x_{a_{2 n}}=0.5$ do both types correspond to the same free energy. For $0.48<x_{a_{2 n}}<0.5$, the clusters rich in component 1 have the lowest free energy, and for $0.5<x_{a_{2 n}}$ $<0.52$, those that are rich in component 2 .

An interesting prediction that was made on the basis of density functional theory by Talanquer et al. ${ }^{12}$ is that when the mixtures exhibit a large miscibility gap in the bulk, cylindrical critical nuclei can appear. The remarkable feature of these nuclei is that the species have (micro) phase separated inside the nucleus. In fact, this type of nucleus has been observed by Clarke et al. ${ }^{19}$ in a computer simulation study of binary liquid Lennard-Jones clusters in vасио. In our simulations, we explored in some detail the possibility of microphase separation in critical clusters. Talanquer and Oxtoby $^{12}$ predict that such clusters should appear for $\Lambda^{*}$ $\geqslant 0.6$ and $x_{a_{2 n}}=0.5$. As discussed above, we find that for $x_{a_{2 n}}=0.5$ and $\Lambda^{*} \geqslant 0.5$, the saddle points that separate the bulk liquid from the metastable vapor correspond to nuclei that are enriched in one of the species. We never found evidence for free-energy saddle points that correspond to cylindrical nuclei. This is not in contradiction to the findings of Clarke et al.: ${ }^{19}$ our simulations do not rule out the possibility that phase-separated clusters form at the top of the barrier separating the two saddle points. However, such clusters then correspond to local free-energy maxima and should play no role in the nucleation process. We have examined the structure of nuclei at the top of the barrier and in the vicinity of $x_{2}=0.5$. For $\Lambda^{*}=0.5$, we found that at the top of the (shallow) ridge separating the two critical nuclei, the species were mixed, rather than phase separated. Even when we "prefabricated" a cylindrical cluster and constrained the cluster at a molar fraction of around 0.5 , we found that after typically $5000 \mathrm{MC}$ cycles the species mixed again, and that the initially cylindrical droplets transform into a spherical shape. For $\Lambda^{*}=0.7$, the situation is slightly more complex. The "unprepared" clusters at the top of the ridge were not cylindrical, although a visual inspection of the snapshots indicated that the species have a strong tendency to phase sepa- rate. Nuclei that were prepared in a phase-separated, cylindrical configuration, remained there for the length of the simulation, provided that the composition of the cluster was kept fixed at $x_{2}=0.5$. However, as soon as the composition of the cluster was allowed to adjust, all phase-separated clusters transformed back into a spherical clusters that were enriched in one of the two species. This illustrates that the cylindrical nuclei correspond to local maxima of the freeenergy surface as a function of the two order parameters, size, and composition, and therefore cannot play a significant role in the nucleation process.

\section{CONCLUSION}

We have developed a new scheme to study critical nuclei involved in gas-liquid nucleation of binary mixtures. Our numerical scheme allows us to compare clusters that have the same barrier height but different compositions. The numerical scheme was found to be both efficient and accurate. Using it, we performed a detailed study of the composition of critical nuclei in binary systems. The results of our simulations support the density functional predictions of Talanquer and Oxtoby ${ }^{12}$ on the nucleation of weakly nonideal mixtures. These mixtures condense via a single type of critical nucleus, in which the components of the mixture can be mixed in all proportions. Depending on the value of the mixing parameter, mixing either enhances or inhibits nucleation.

For the nucleation of mixtures that show a macroscopic miscibility gap, the picture that emerges is more complex. We find that nucleation in these systems can still be initiated by clusters in which both species are mixed. However, when the cluster size increases, the path bifurcates and two channels develop, corresponding to nuclei enriched in one of the two components. In all cases, the critical nuclei are found to have a spherical shape. The cylindrical, microphase separated nuclei predicted by Talanquer and Oxtoby ${ }^{12}$ only appear at the top of the free-energy ridge separating the two types of critical nuclei. The cylindrical clusters do not correspond to saddle points and they probably play no role in the nucleation of immiscible mixtures.

The cluster size at which the bifurcation of the path occurs depends on the value of the mixing parameter. Hence, whether or not one or two types of critical nuclei can be formed depends on the supersaturation and the value of the mixing parameter. For larger values of $\Lambda^{*}$, larger supersaturations, corresponding to smaller critical-nucleus sizes, are required in order to find mixed critical nuclei. Correspondingly, when the nucleation process is dominated by one type of critical cluster at a given supersaturation, it can proceed via two types of nuclei at smaller supersaturation. This also implies that, in experiments on the nucleation behavior of partially miscible or immiscible mixtures, care should be taken when deriving the composition of the critical nuclei from the variation of the nucleation rate with supersaturation. ${ }^{5}$ Our simulations suggest that the measured average composition of the nucleus need not be the most likely one. 


\section{ACKNOWLEDGMENTS}

This work was supported in part by "Scheikundig Onderzoek Nederland" (SON) with financial aid from NWO ("Nederlandse Organisatie voor Wetenschappelijk Onderzoek"). The work of the FOM Institute is part of the research program of "Stichting Fundamenteel Onderzoek der Materie" (FOM) and is supported by NWO. P. R. ten Wolde would like to thank David Oxtoby for the stimulating discussions. We also thank Alfons van Blaaderen and James Polson for a critical reading of the manuscript.

${ }^{1}$ Y. Viisanen, R. Strey, and H. Reiss, J. Chem. Phys. 99, 4680 (1993).

${ }^{2}$ R. Strey and Y. Viisanen, J. Chem. Phys. 99, 4693 (1993).

${ }^{3}$ Y. Viisanen, R. Strey, A. Laaksonen, and M. Kulmala, J. Chem. Phys. 100, 6062 (1994).

${ }^{4}$ R. Strey, Y. Viisanen, and P. E. Wagner, J. Chem. Phys. 103, 4333 (1995).

${ }^{5}$ Y. Viisanen, P. E. Wagner, and R. Strey, J. Chem. Phys. 108, 4257 (1998).
${ }^{6}$ K. N. H. Looijmans, C. C. M. Luijten, G. C. J. Hofmans, and M. E. W. van Dongen, J. Chem. Phys. 102, 4531 (1995).

${ }^{7}$ K. N. H. Looijmans, C. C. M. Luijten, and M. E. W. van Dongen, J. Chem. Phys. 103, 1714 (1995).

${ }^{8}$ R. M. Nyquist, V. Talanquer, and D. W. Oxtoby, J. Chem. Phys. 103, 1175 (1995)

${ }^{9}$ D. W. Oxtoby and D. Kashchiev, J. Chem. Phys. 100, 7665 (1994).

${ }^{10}$ A. Laaksonen and D. W. Oxtoby, J. Chem. Phys. 102, 5803 (1995).

${ }^{11}$ A. Laaksonen and D. W. Oxtoby, J. Chem. Phys. 106, 7268 (1997).

${ }^{12}$ V. Talanquer and D. W. Oxtoby, J. Chem. Phys. 104, 1993 (1996).

${ }^{13}$ P. R. ten Wolde and D. Frenkel J. Chem. Phys. 109, 9901 (1998), this issue.

${ }^{14}$ G. M. Torrie and J. P. Valleau, Chem. Phys. Lett. 28, 578 (1974).

${ }^{15}$ D. A. Kofke, J. Chem. Phys. 98, 4149 (1993).

${ }^{16}$ P. Bolhuis and D. Frenkel, J. Chem. Phys. 106, 666 (1997).

${ }^{17}$ D. Frenkel and B. Smit, Understanding Molecular Simulation, From Algorithms to Applications (Academic, San Diego, 1996).

${ }^{18}$ B. Smit, J. Chem. Phys. 96, 8639 (1992).

${ }^{19}$ A. S. Clarke, R. Kapral, and G. N. Patey, J. Chem. Phys. 101, 2432 (1994).

${ }^{20}$ D. W. Oxtoby and A. Laaksonen, J. Chem. Phys. 102, 6846 (1995).

${ }^{21}$ A. K. Ray, M. Chalam, and L. K. Peeters, J. Chem. Phys. 85, 2161 (1986). 